LA- - 11502-MS

DE89 011120

Parameterization of Pion Production and Reaction Cross Sections at LAMPF Energies

R. L. Burman

E.S.Smith*

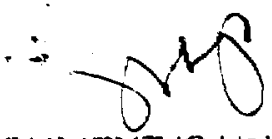




\section{CONTENTS}

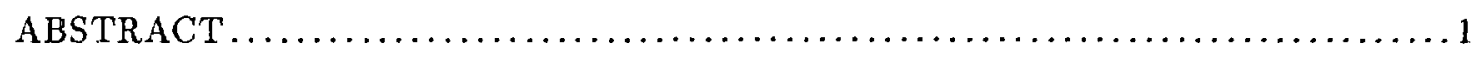

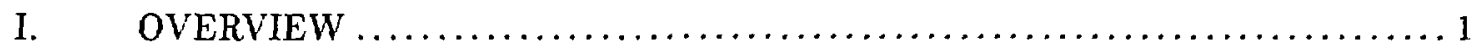

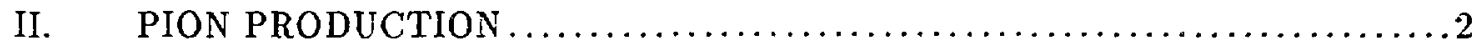

III. PION REACTION CROSS SECTIONS $\ldots \ldots \ldots \ldots \ldots \ldots \ldots \ldots \ldots \ldots \ldots \ldots \ldots$

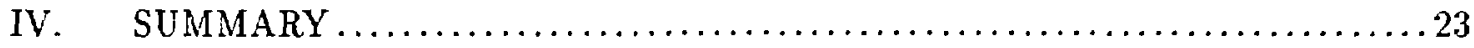




\section{TABLES}

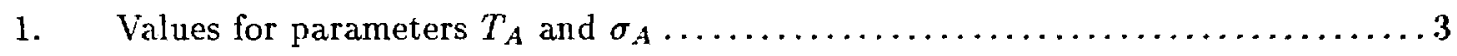

2. Values for total cross-section parameters $\ldots \ldots \ldots \ldots \ldots \ldots \ldots \ldots \ldots \ldots \ldots \ldots$

\section{FIGURES}

1. Basis splines for the parameterization of $A m p(\theta) \ldots \ldots \ldots \ldots \ldots \ldots \ldots \ldots \ldots \ldots \ldots \ldots \ldots \ldots$

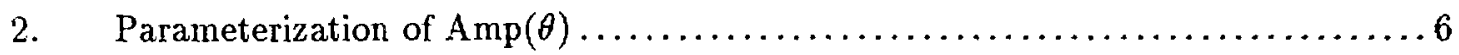

3. Energy dependence of the total pion production cross section ............. 8

4. $\pi^{+}$production from hydrogen, $T_{p}=585 \mathrm{MeV} \ldots \ldots \ldots \ldots \ldots \ldots \ldots \ldots \ldots$

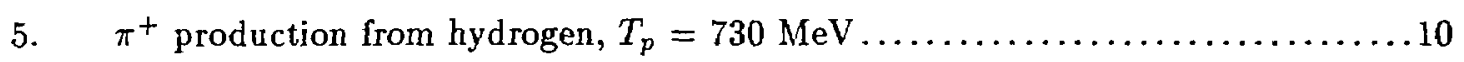

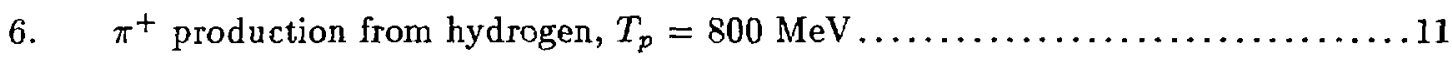

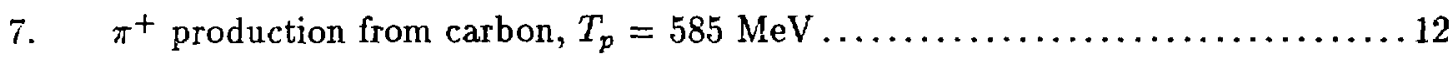

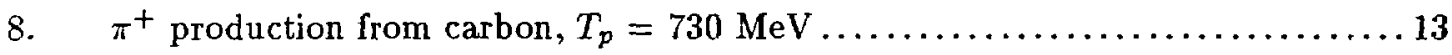

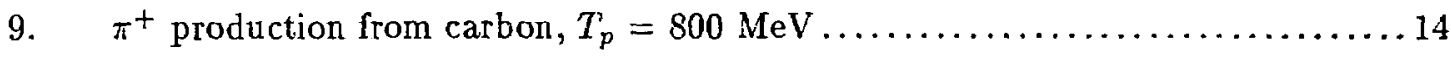

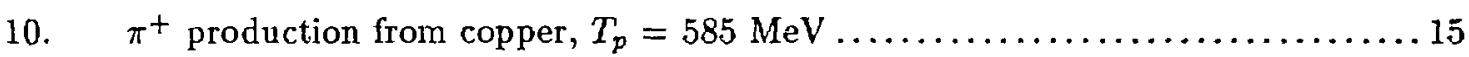

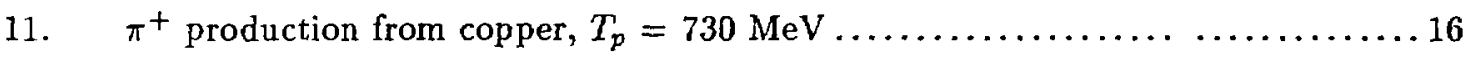

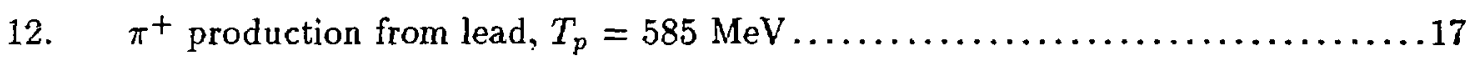

13. $\pi^{+}$production from lead, $T_{p}=730 \mathrm{MeV} \ldots \ldots \ldots \ldots \ldots \ldots \ldots \ldots \ldots \ldots \ldots \ldots \ldots \ldots \ldots \ldots \ldots \ldots$

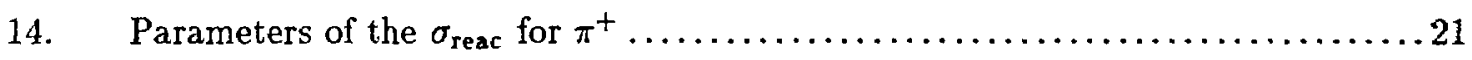

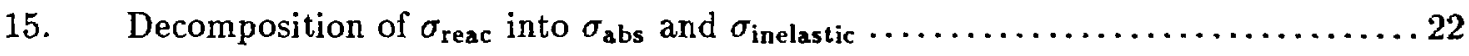

16. Comparison of the parameterization of $\sigma_{\text {reac }}$ with data $\ldots \ldots \ldots \ldots \ldots \ldots \ldots 23$ 


\title{
PARAMETERIZATION OF PION PRODUCTION AND REACTION CROSS SECTIONS AT LAMPF ENERGIES
}

\author{
by
}

\author{
R. L. Burman and E. S. Smith
}

\begin{abstract}
A parameterization of pion production and reaction cross sections is developed for event ual use in modeling neutrino production by protons in a beam stop. Emphasis is placed upon smooth parameterizations for proton energies up to $800 \mathrm{MeV}$, for all pion energies and angles, and for a wide range of materials. The resulting representations of the data are well-behaved and can be used for extrapolation to regions where there are no measurements.
\end{abstract}

\section{OVERVIEW}

In order to model the production of neutrinos from the LAMPF beam stop, we require knowledge of pion production by protons and pion reaction cross sections over a broad range of all kinematic variables involved and for many materials. Our general philosophy in modeling the neutrino production is to emphasize smooth and simple parameterizations, which can be used over a wide range of energies, angles and materials at the expense of not reproducing the measured pion cross sections exactly. This model of production and decay is then compared with data that directly measure the distribution of pion decays inside the beam stop. ${ }^{1}$ The parameters of the model are then tuned, within reasonable limits, to reproduce these data. The Monte Carlo program, which uses this parameterization to model the neutrino flux, will be described elsewhere. ${ }^{2}$ For the present paper we confine ourselves to the form of the parameterization used as input to the Monte Carlo program. No tuning of parameters has yet been made.

The proton beam incident on the LAMPF beam stop has a typical energy of $775 \mathrm{MeV}$.* As the proton beam is absorbed in the beam stop, pions are produced all along the proton track. Pions come to rest in a typical proper time of $4 \times 10^{-10} \mathrm{~s}$, allowing $\sim 2 \%$ to decay in flight. Negative pions at rest are immediately absorbed into the nucleus from atomic orbitals and only positive pions decay. Thus, the beam-stop neutrino source is

* The proton energy exiting the Linac is $800 \mathrm{MeV}$. The two pion production targets $\mathrm{A1}$ and $A 2$, with usual thicknesses of 3.0 and $4.0 \mathrm{~cm}$ of $A T J$ graphite, reduce the beam energy by $25 \mathrm{MeV}$. 
primarily due to the decay of positive pions and unpolarized* muons at rest, which decay isotropically. It is therefore important to model pion production down to zero pion kinetic energy, a region difficult to measure and largely unimportant for many applications. The most couplete set of pion production cross sections was measured for protons of $730-\mathrm{MeV}$ incident kinetic energy. ${ }^{3}$ The differential cross sections were measured over a wide range of pion energies $\left(T_{\pi}=30-553 \mathrm{MeV}\right)$ and scattering angles $\left(\theta=15-150^{\circ}\right)$ for many targets. A second experimental survey was taken for protons with incident energy of $585 \mathrm{MeV}$. $^{4}$ This experiment did not cover the dynamic range of the former, but was fairly complete nevertheless.

The LAMPF beam stop proper is water-cooled copper, but protons also interact in the isotope-production stringers, which contain various amounts of heavy materials (e.g. Cesium and Europium) that vary according to the demand for radioisotopes. ${ }^{5}$ The beam stop of the spallation neutron source ISIS of the Rutherford Appleton Laboratory is made of uranium. ${ }^{6}$ It is, therefor,$r^{\prime}$ general interest to have parameterizations that can be used for all targets.

As pions slow down $i_{h_{1}}$ the beam stop, they may be absorbed or scattered, which changes both the energy and angular spectrum of the original pions. A typical absorption or inelastic-scattering cross section is $500 \mathrm{mb}$ (i.e., $\sigma_{\text {reac }}=\sigma_{\text {abs }}+\sigma_{\text {inelastic }} \sim 1$ b). This corresponds to a scattering length of $100 \mathrm{~g} / \mathrm{cm}^{2}$ or $10 \mathrm{~cm}$ in copper. Thus, approximately half of the produced pions interact strongly before they decay and affect the neutrino spectrum accordingly. Measurements of the pion-reaction cross section, which includes both absorption and inelastic scattering, are not extensive and rely on a variety of experiments to extract their result. ${ }^{7}$ Thus, it is difficult to properly account for the various systematic errors.

\section{PION PRODUCTION}

There exist two measurements of pion-production cross sections, at proton kinetic energies of $T_{p}=585$ and $730 \mathrm{MeV},{ }^{4,3}$ that have covered a wide variety of nuclei as well as a broad range of produced pion energies $\left(T_{\pi}\right)$ and angles $(\theta)$. We, therefore, use their data as a basis for our parameterization and use other measurements. ${ }^{8,9,10}$ only for various checks. The doubly differential positive-pion cross sertion for a given material $Z$ and fixed proton kinetic energy $T_{p}$ is written in the following form:

$$
\frac{d^{2} \sigma}{d \Omega_{\pi} d T_{\pi}}=\operatorname{Amp}(\theta) e^{-\left(\frac{\bar{T}-T_{x}}{\sqrt{2} \sigma(\theta)}\right)^{2}} \times\left(1+e^{\frac{T_{x}-T_{E}}{B}}\right)^{-1}(\mu \mathrm{b} / \mathrm{MeV} / \mathrm{sr})
$$

This approximation of the energy dependence by a Gaussian function is similar to that used by Dombeck ${ }^{11}$ in a calculation of neutrino fluxes from in-flight pion decay. The parameters $\operatorname{Amp}(\theta), \bar{T}$, and $\sigma$ were obtained by fitting the data in references ${ }^{3,4}$ using standard minimization techniques, but weighting all points equally, at the two proton energies $T_{p}$ $=585$ and $730 \mathrm{MeV}$ for hydrogen, carbon, copper, and lead. These values have in turn

\footnotetext{
* Integrated over the spectrum.
} 
been expressed in as simple a form as possible to cover the entire range of variables in the problem. The functional dependence on $T_{\pi}$ is simple, it appears only in the exponent of the Gaussian and in the high-energy cut-off factor. Simple analytic forms suffice for the angle dependence of the Gaussian energy parameters $\bar{T}$ and $\sigma$ :

$$
\begin{aligned}
\bar{T}(\theta) & =48+330 e^{-\left(\frac{\theta}{T_{A}}\right)} \\
\sigma(\theta) & =\sigma_{A} e^{-\left(\frac{\theta}{85}\right)} \\
T_{A}\left(Z, T_{p}\right) & =\frac{T_{A}(Z, 730)\left(T_{p}-585\right)-T_{A}(Z, 585)\left(T_{p}-730\right)}{730-585} \\
\sigma_{A}\left(Z, T_{p}\right) & =\frac{\sigma_{A}(Z, 730)\left(T_{p}-585\right)-\sigma_{A}(Z, 585)\left(T_{p}-730\right)}{730-585}
\end{aligned}
$$

Here, the secondary parameters $T_{A}$ and $\sigma_{A}$ are assumed to have a piece-wise linear dependence on proton energy $T_{p}$ and to have constant values over step-wise ranges in atomic number $Z$. The values for $T_{A}$ and $\sigma_{A}$ over the appropriate $Z$ range are given in Table I.

Table I. Values for Parameters $T_{A}$ and $\sigma_{A}$ at $T_{p}=585,730 \mathrm{MeV}$ and for Ranges in $Z$.

\begin{tabular}{cccc}
\hline Proton Energy & Atomic Number & $T_{A}$ & $\sigma_{A}$ \\
\hline $585 \mathrm{MeV}$ & 1 & 64.5 & 155 \\
& $2-8$ & 28.9 & 130 \\
& $8-92$ & 26.0 & 135 \\
\hline $730 \mathrm{MeV}$ & 1 & 53.0 & 127 \\
& $2-8$ & 34.2 & 150 \\
& $8-92$ & 29.9 & 166 \\
\hline
\end{tabular}

The dependence of the amplitude $\operatorname{Amp}(\theta)$ on material and proton energy as well as scattering angle is nontrivial, so we have chosen to parameterize it with $B$-splines ${ }^{12}$ over the allowed angular region $\left(0 \leq \theta \leq 180^{\circ}\right)$ :

$$
\begin{aligned}
\operatorname{Amp}(\theta) & =\operatorname{Norm}(Z) \times \sum_{n=1}^{5} a_{n} B_{n} \\
a_{1} & =\min \left[27-4\left(\frac{730-T_{p}}{730-585}\right)^{2}, 27\right] \\
a_{2} & =18.2 \\
a_{3} & =8 \\
a_{4} & =13+(Z-12) / 10 \\
a_{5} & =9+(Z-12) / 10-\left(T_{p}-685\right) / 20
\end{aligned}
$$




$$
\begin{aligned}
\operatorname{Norm}(Z) & =\sum_{m=0}^{3} c_{m}(\operatorname{In} Z)^{m} Z^{1 / 3} \\
c_{0} & =0.8851 \\
c_{1} & =-0.1015 \\
c_{2} & =0.1459 \\
c_{3} & =-0.0265
\end{aligned}
$$

Pion production from hydrogen requires some modification of the parameters listed above: the coefficients $a_{1}, a_{2}, a_{4}$, and $a_{5}$ are doubled in order to reproduce the measured angular distributions.

We digress for a moment to make some general comments about $B$-splines, as many people are not familiar with them. Splines are piece-wise continuous polynomials defined on some specified interval. Parabolic splines, the ones used in this paper, are polynomials of second degree, which assure well defined functional behavior within the interval of interest. Parabolas are defined between points on the interval called knots, where they are joined together by continuity conditions. For example, the splines used to specify $\operatorname{Amp}(\theta)$ have a knot sequence of $\left(0,0,0,30,70,180,180,180^{\circ}\right)$ and are shown in Fig. 1. For this set of splines, the second derivative is allowed to be discontinuous at 30 and $70^{\circ}$. The function itself is allowed to be discontinuous at 0 and $180^{\circ}$. If one of the knots had been specified twice, then the splines would have a discontinuous first derivative, which we do not allow. The knot sequence was chosen in such a way that the splines could easily be molded to fit the functional form of $A m p(\theta)$ (see Fig. 2).

The splines represented on the angular interval are decomposed into a basis, called $B$-splines, which is normalized so that

$$
\sum_{n=1}^{N} B_{n}=1 .
$$

The $B$-splines are completely specified by the interval, the knot sequence and the degree of their polynomials. Any function on this interval that matches the continuity conditions of the splines, e.g. $A m p(\theta)$, can then be written as a linear combination of the $B$-splines. In this case, the coefficients of the expansion $\left(a_{n}\right)$ are used to specify the continuous behavior of $\operatorname{Amp}(\theta)$ as a function of atomic number $Z$ and proton energy $T_{p}$ as given above. This parameterization is very flexible and allows one to systematically vary the amplitude of the cross section at any angle of interest. For example, one may easily tune the amplitude at $0^{\circ}$ by changing the coefficient $a_{1}$. The parameterization of the amplitude $\operatorname{Amp}(\theta)$ is shown in Fig. 2 compared to the values obtained by least-squares fits to the data. The normalization factor Norm has been adjusted to give the measured total cross section at $T_{p}=730 \mathrm{MeV}$. However, total cross sections are handled separately for convenience, as indicated below.

The high-energy cut-off function is intended to take account of kinematical factors, which inhibit pion production in hydrogen or at forward angles. Hydrogen is treated as a special case, and the mean cut-off $T_{F}$ is taken to be the pion energy in the reaction 


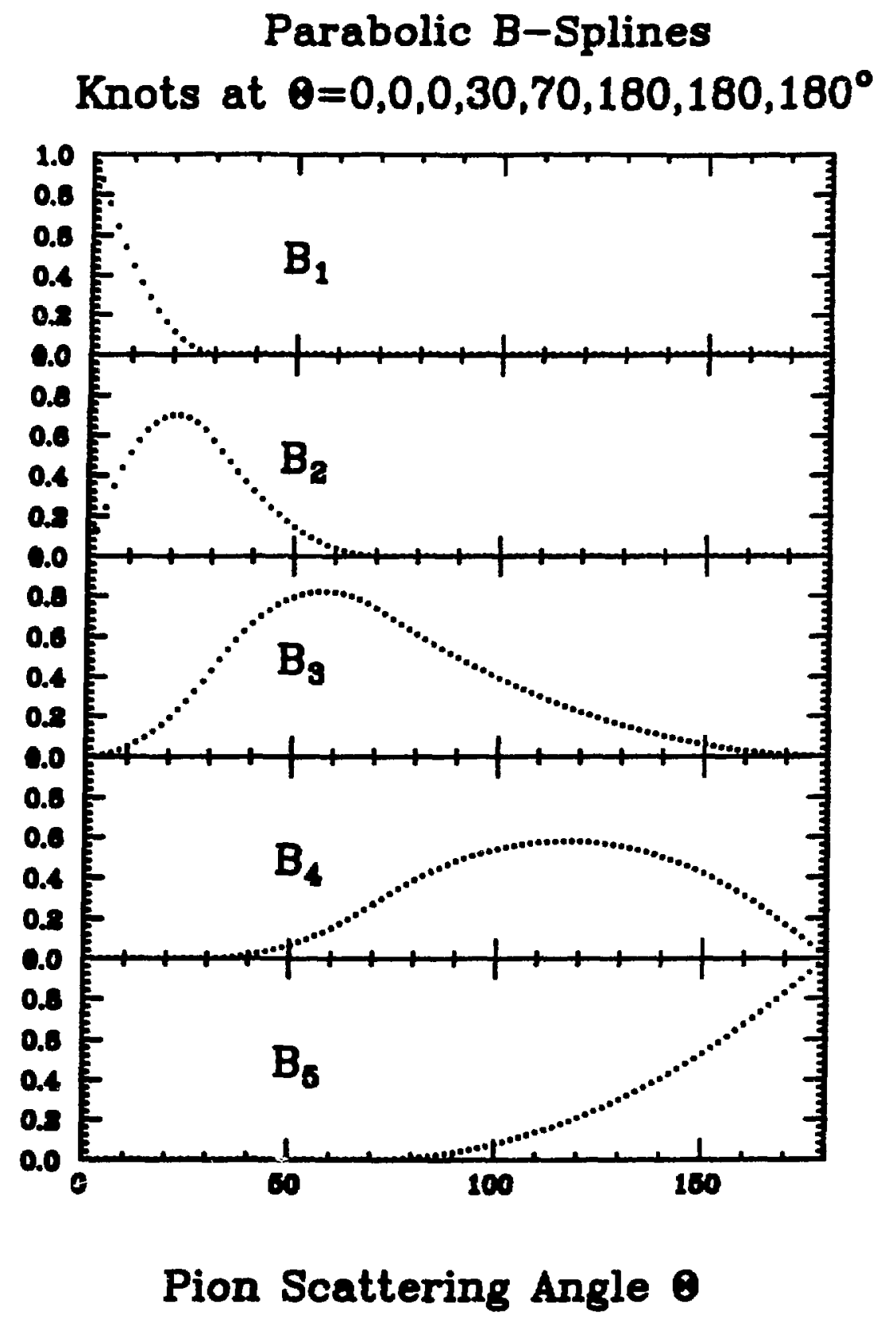

Fig. 1. Basis splines for the parameterization of $\operatorname{Amp}(\theta)$ of the differential pion production cross section. 


\section{Amplitude of Cross Section ( $\mu \mathrm{b})$}

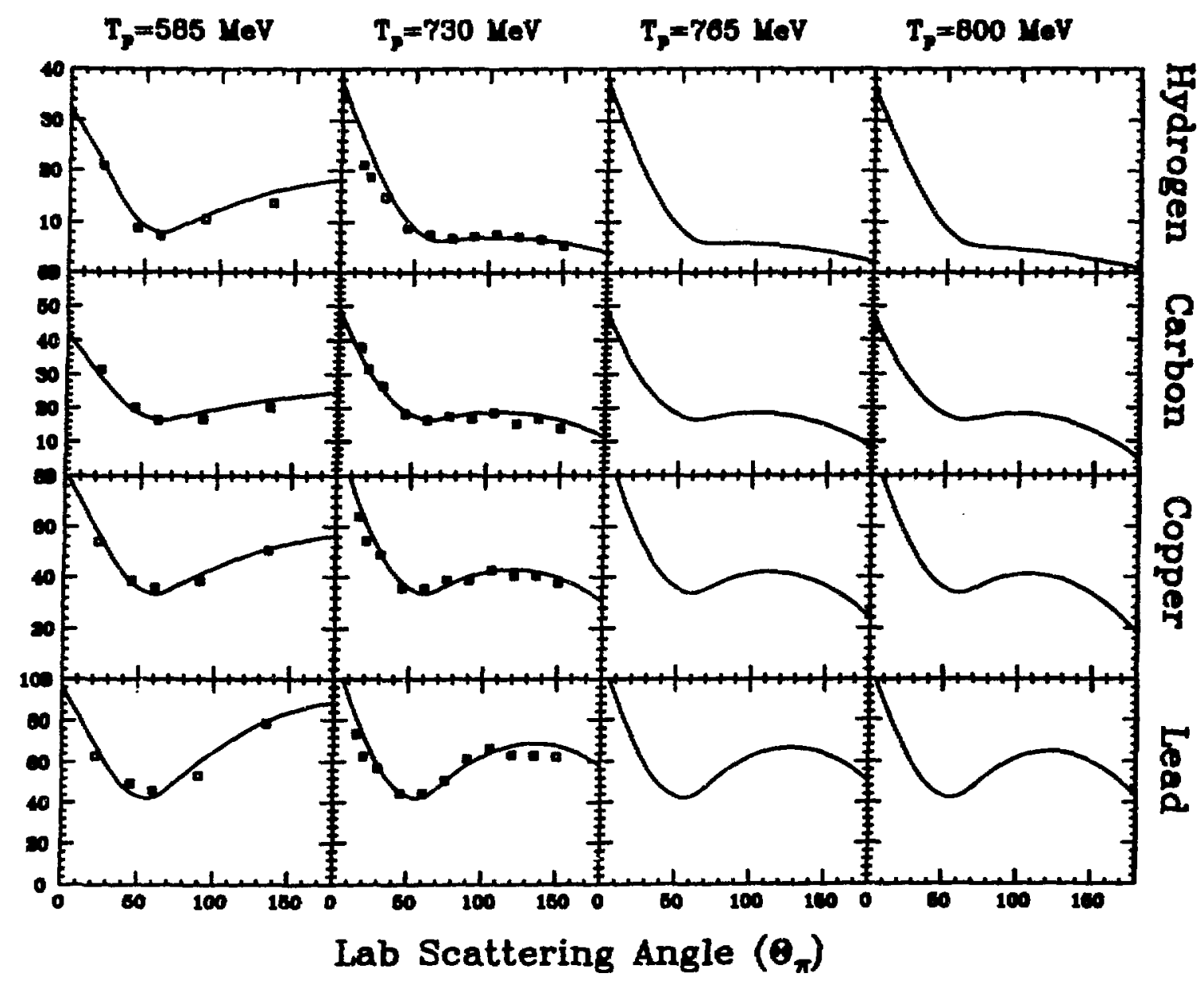

Fig. 2. The parameterization of $\operatorname{Amp}(\theta)$ is shown superimposed on the fitted values of the parameter. The curves at $T_{p}=765$ and $800 \mathrm{MeV}$ show the parameterization extended to higher energies where no data are available.

$p p \rightarrow \pi^{+} d$. For other nuclei, the cut off is used to insure energy conservation $\left(T_{F}=T_{p}-\right.$ $140 \mathrm{MeV}-2 \mathrm{~B})$. Resolution determines the sharpness of the cut off, which is controlled by the parameter $B$ currently set to $25 \mathrm{MeV}$.

The total cross section obtained by integration of the parameterization of the differential cross section reproduces the published cross sections to $\sim 10 \%$. The absolute cross section is handled separately to allow flexibility in understanding the effects of overail normalization. For the moment we assume the total cross section is a linear function of $T_{p}$. Above $T_{p}=585 \mathrm{MeV}$ the curve is determined by the quoted cross sections at $T_{p}=730$ and $585 \mathrm{MeV}$ from references. ${ }^{3,4}$ Below the assumed break point of $T_{p}=585 \mathrm{MeV}$, the total 
cross section decreases linearly to zero at $T_{p}=325 \mathrm{MeV}$. ${ }^{*}$ This behavior is shown in Fig. 3 . Measured cross sections from Refs. 3 and 4 are used to determine the pion production total cross-section dependence on atomic number $Z$. A $Z^{1 / 3}$ dependence is used, with fit coeffcients from Refs. 3 and 4 . Below $Z=12$, an additional factor linear in $Z$ is employed to better fit the data down to helium; as for the doubly differential cross sections, pion production on hydrogen is a special case. The algorithms used to calculate the $Z$ dependence are:

$$
\begin{aligned}
\sigma(Z>11) & =\sigma_{0}\left(T_{p}\right) Z^{1 / 3} \\
\sigma(1<Z<12) & =\sigma_{12}\left(T_{p}\right)(Z / 6)^{1 / 3}(0.77+0.039 Z) \\
\sigma(Z=1) & =\sigma_{1}\left(T_{p}\right) .
\end{aligned}
$$

The coefficients as a function of proton energy $T_{p}$ are given in Table II.

Table II. Values for Total Cross-Section Parameters at $T_{p}=585,730 \mathrm{MeV}$.

\begin{tabular}{crrr}
\hline$T_{p}$ & $\sigma_{0}$ & \multicolumn{1}{c}{$\sigma_{1}$} & $\sigma_{12}$ \\
\hline $585 \mathrm{MeV}$ & 19.65 & 9.70 & 28.5 \\
$730 \mathrm{MeV}$ & 24.50 & 13.50 & 35.0 \\
& & & \\
\hline
\end{tabular}

Our parameterization of pion production is compared to the data in Refs. $3,4,8$, and 9 in Figs. 4-13. The data at $T_{p}=730 \mathrm{MeV}$ have been relied on most heavily because they are the most extensive. The parameterization, therefore, reproduces these data quite well. The agreement for hydrogen is good, given that special kinematical considerations apply. The curves systematically underestimate the data for $T_{p}=585 \mathrm{MeV}$ at about $50^{\circ}$ and overestimate the data for $T_{p}=730 \mathrm{MeV}$ and $20^{\circ}$. These conditions indicate either limitations in the basic form of the parameterization or flaws in the data because they do not represent smooth variations as a function of proton energy and scattering angle. Cross checks of cross sections predicted at higher energies and forward angles are shown in Figs. 6 and 9, where the extrapolations are compared to data from Refs. 8 and 9 , which were not used to fix any parameters. On the whole, this representation of pion production is convincing and predicts the cross sections for all materials, proton energies below $800 \mathrm{MeV}$, and all production angles.

\section{PION-REACTION CROSS SECTIONS}

The interaction of pions in the beam stop is the sum of elastic, inelastic, and absorption processes. Elastic scattering accounts for about a third of the total cross section. However, for pion energies above $T_{\pi}=100 \mathrm{MeV}$, elastic scattering is confined to a cone less than

* The total cross section has dropped by two orders of magnitude at this point. This can be estimated from the fluxes of secondary pion beams at the Rochester cyclotron, produced from an internal $240-\mathrm{MeV}$ proton beam. ${ }^{14}$ See also Ref. 22. 


\section{Total Cross Sections for $\pi^{+}$Production}

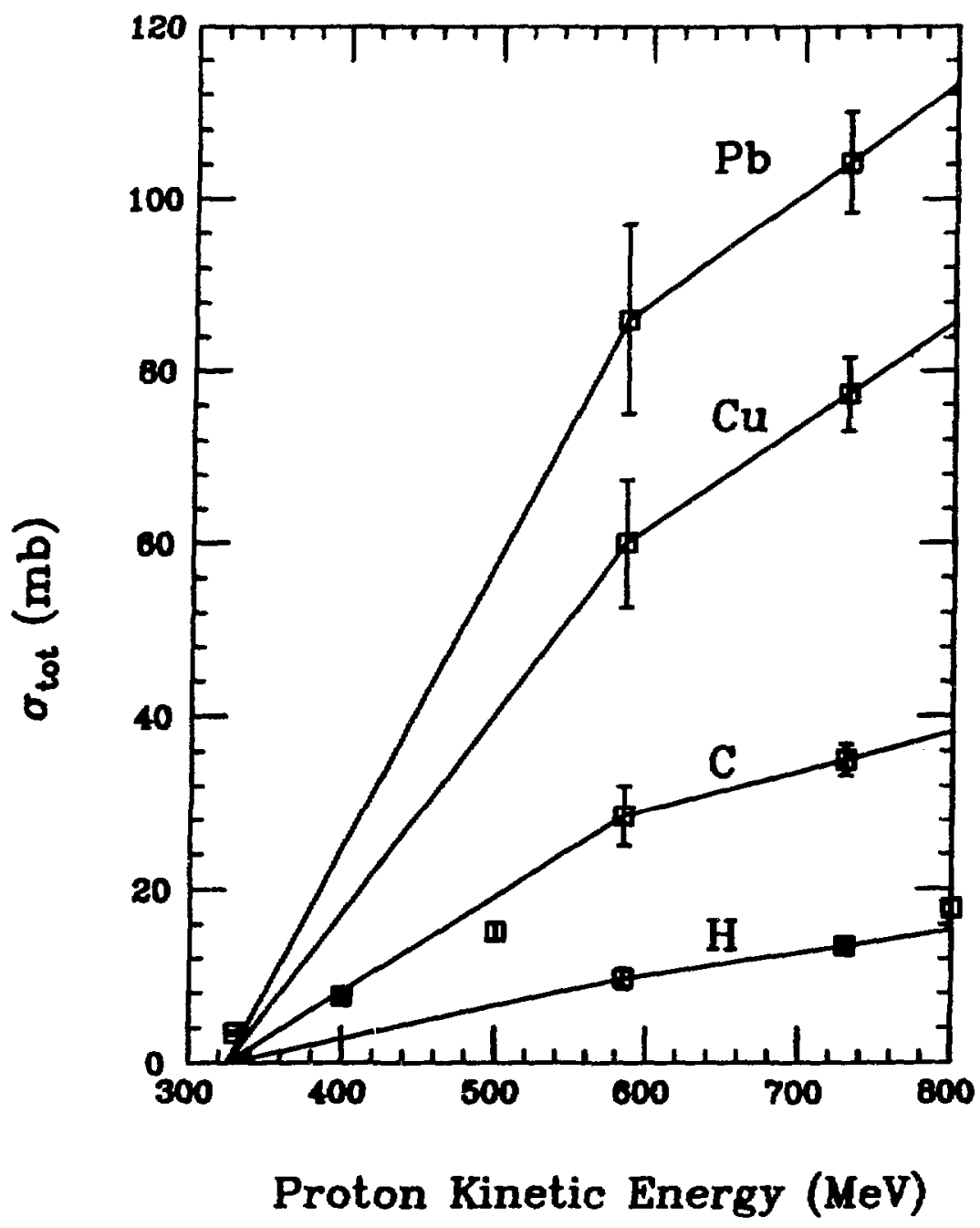

Fig. 3. We show the assumed dependence of the total pion-production cross section on proton energy $T_{p}$. The data points are from Refs. 3, 4, 13, and 21. Also see previous footnote. 


\section{Inclusive $\pi^{+}$Production from Hydrogen $\left(\mathrm{T}_{\mathrm{p}}=585 \mathrm{MeV}\right)$}

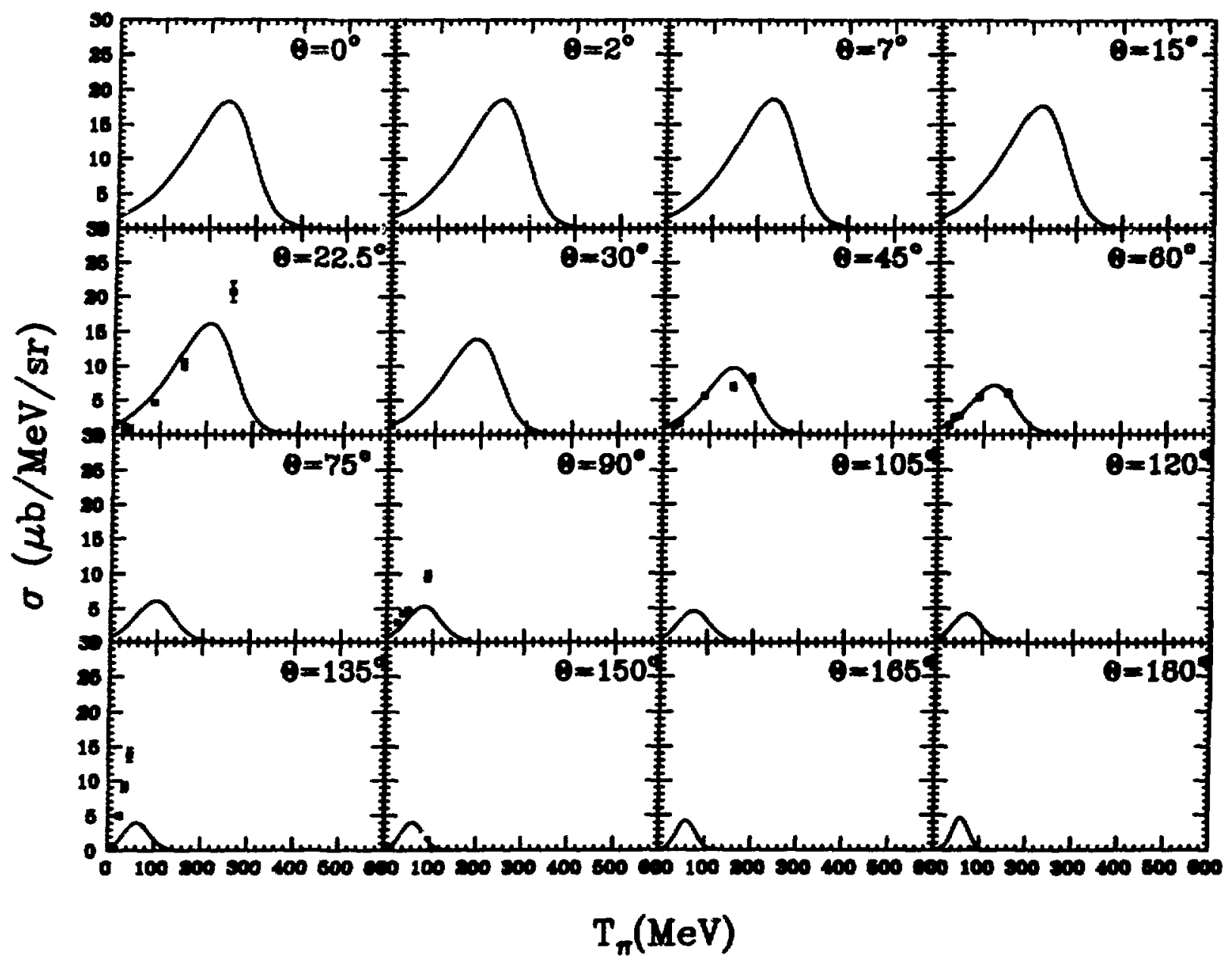

Fig. 4. The parameterization for $\pi^{+}$production from hydrogen is compared with the data from Ref. 4. 


\section{Inclusive $\pi^{+}$Production from Hydrogen $\left(T_{p}=730 \mathrm{MeV}\right)$}

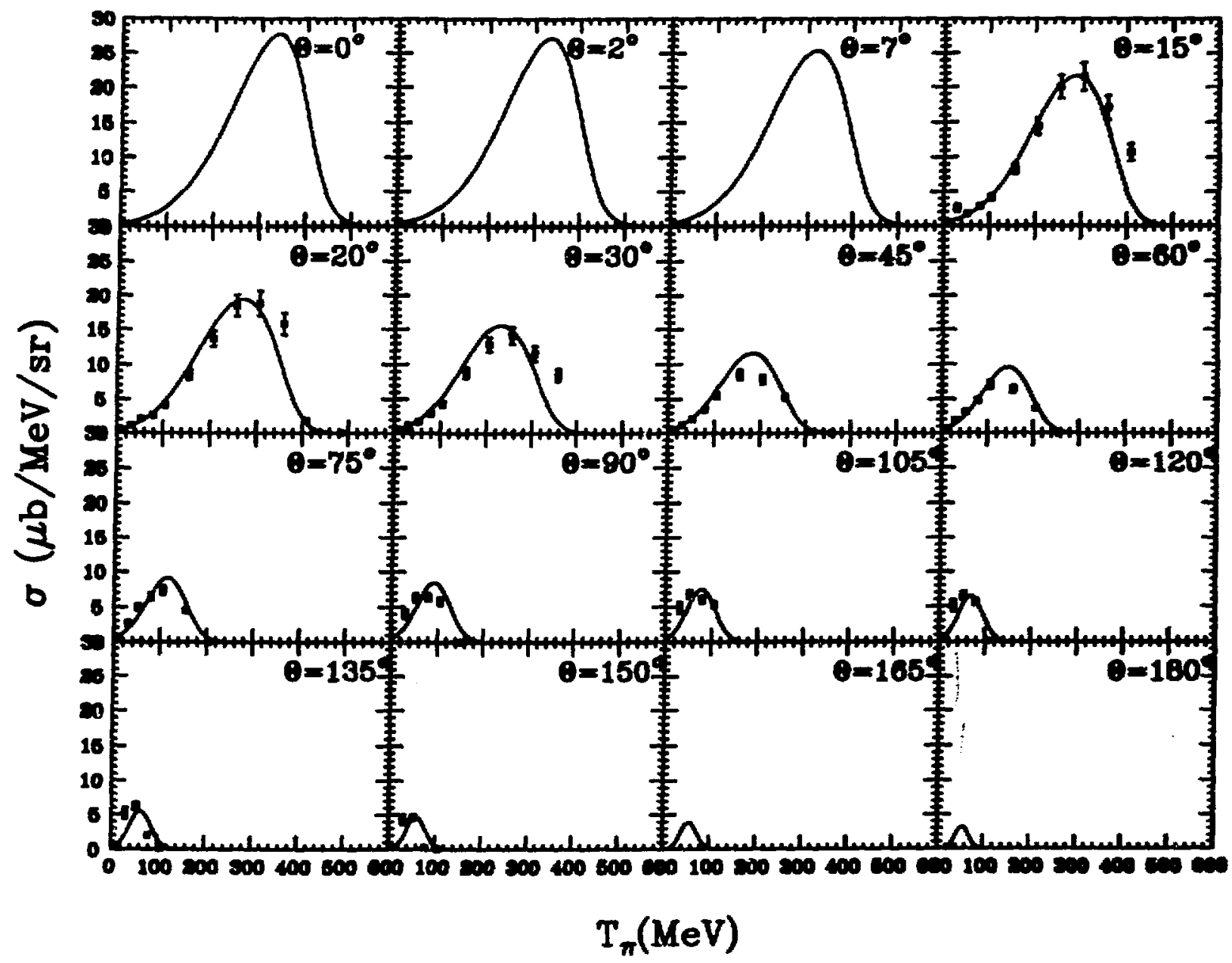

Fig. 5. The parameterization for $\pi^{+}$production from hydrogen is compared with the data from Ref. 3. 
Inclusive $\pi^{+}$Production from Hydrogen $\left(\mathrm{T}_{\mathrm{p}}=800 \mathrm{MeV}\right)$

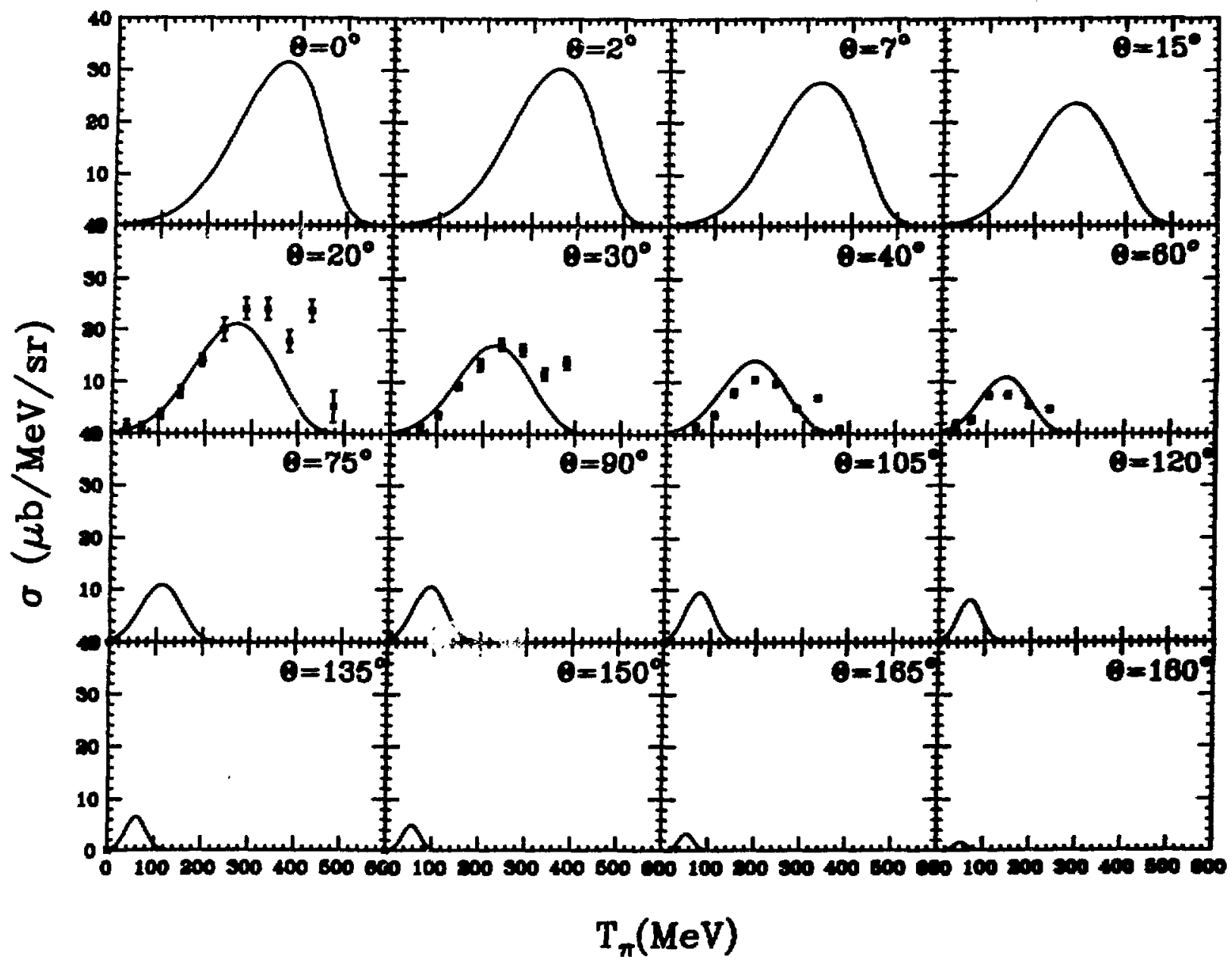

Fig. 6. The parameterization for $\pi^{+}$production from hydrogen is compared with the data from Ref. 9. These data are a test of the parameterization, as they were not used to fix any parameters. This experiment is able to resolve the kinematic peak of $p p \rightarrow \pi^{+} d$. 


\section{Inclusive $\pi^{+}$Production from Carbon $\left(T_{p}=585 \mathrm{MeV}\right)$}

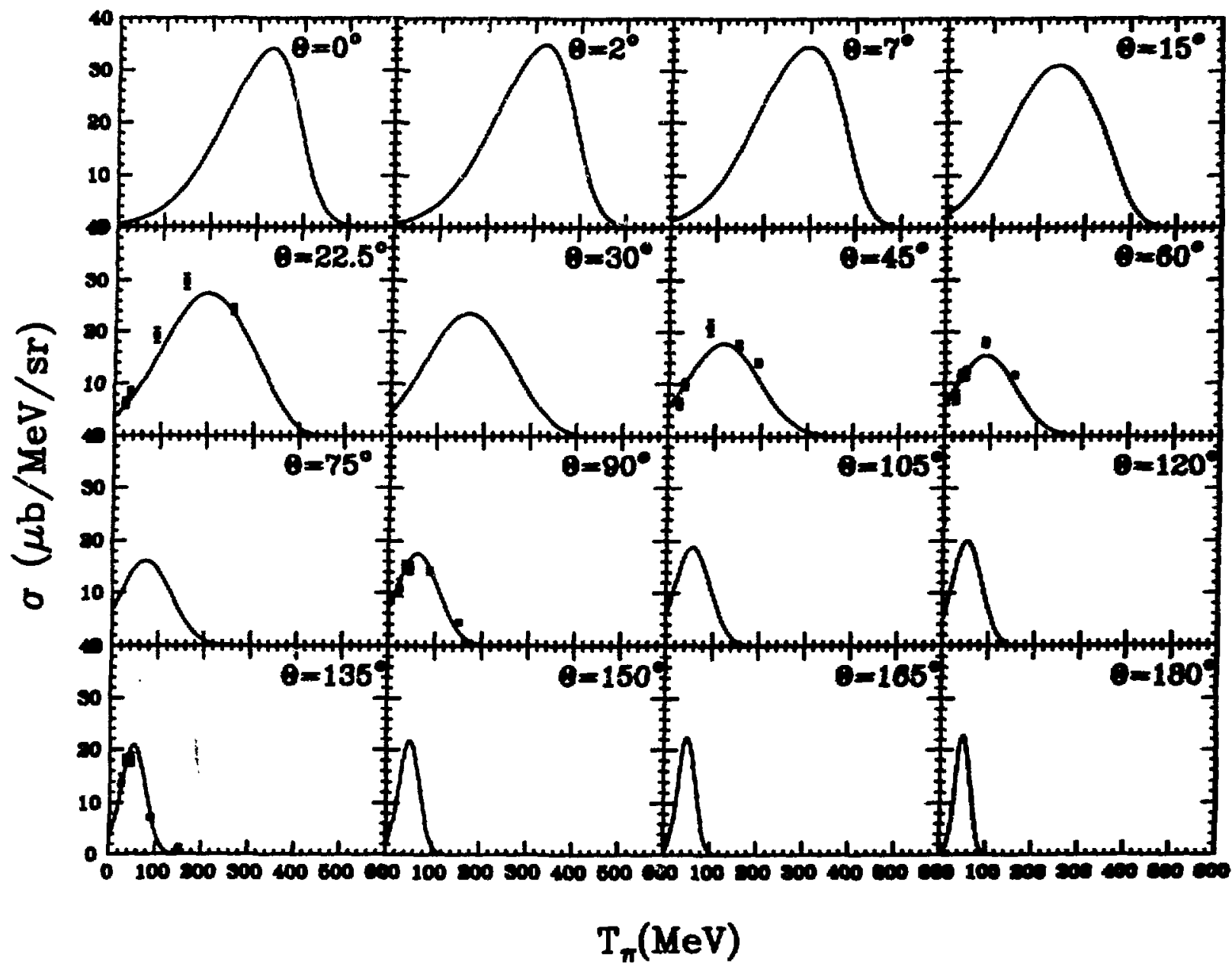

Fig. 7. The parameterization for $\pi^{+}$production from carbon is compared with the data from Ref. 4. 
Inclusive $\pi^{+}$Production from Carbon $\left(\mathrm{T}_{\mathrm{p}}=730 \mathrm{MeV}\right)$

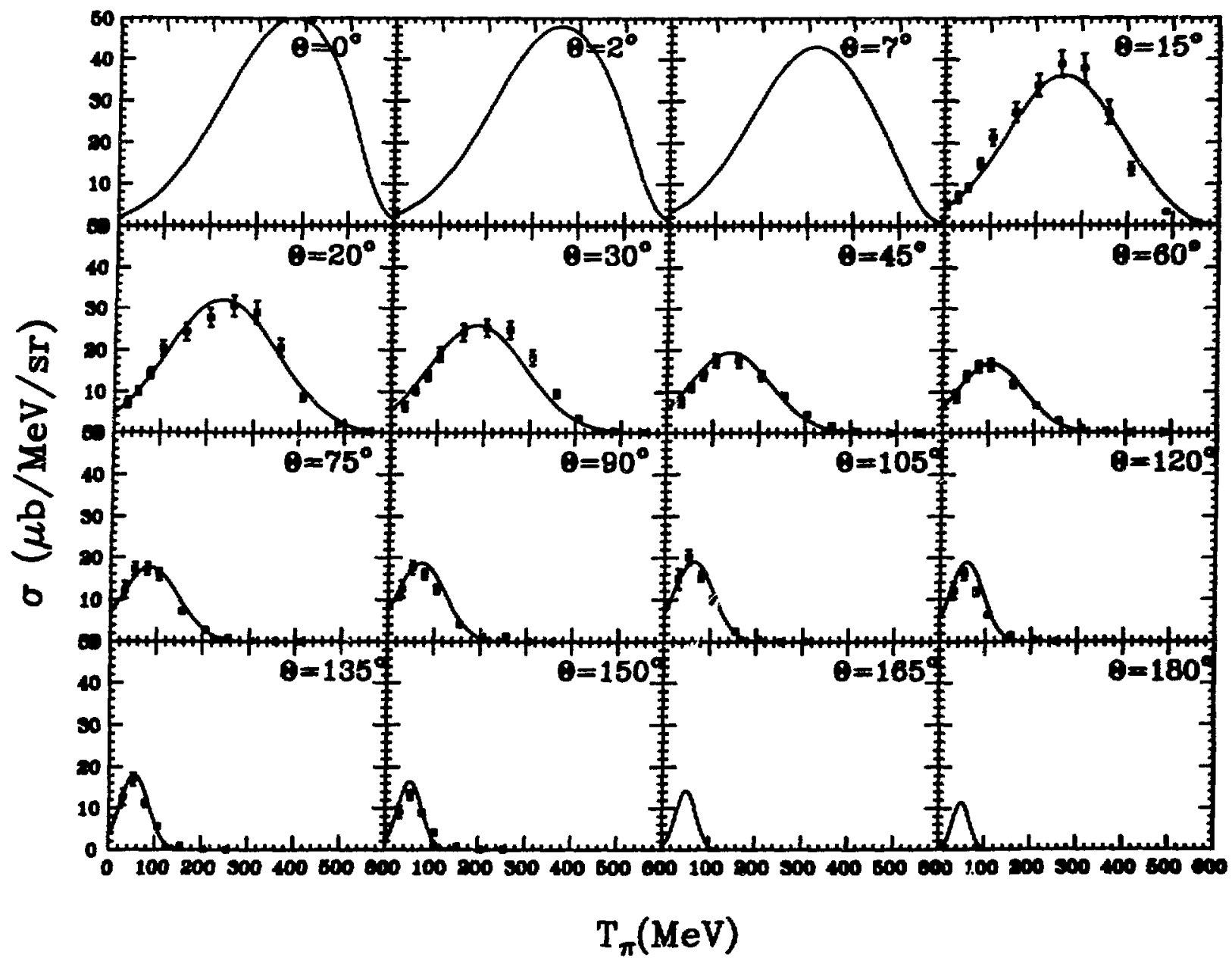

Fig. 8. The parameterization for $\pi^{+}$production from carbon is compared with the data from Ref. 3. 
Inclusive $\pi^{+}$Production from Carbon $\left(\mathrm{T}_{\mathrm{p}}=800 \mathrm{MeV}\right)$

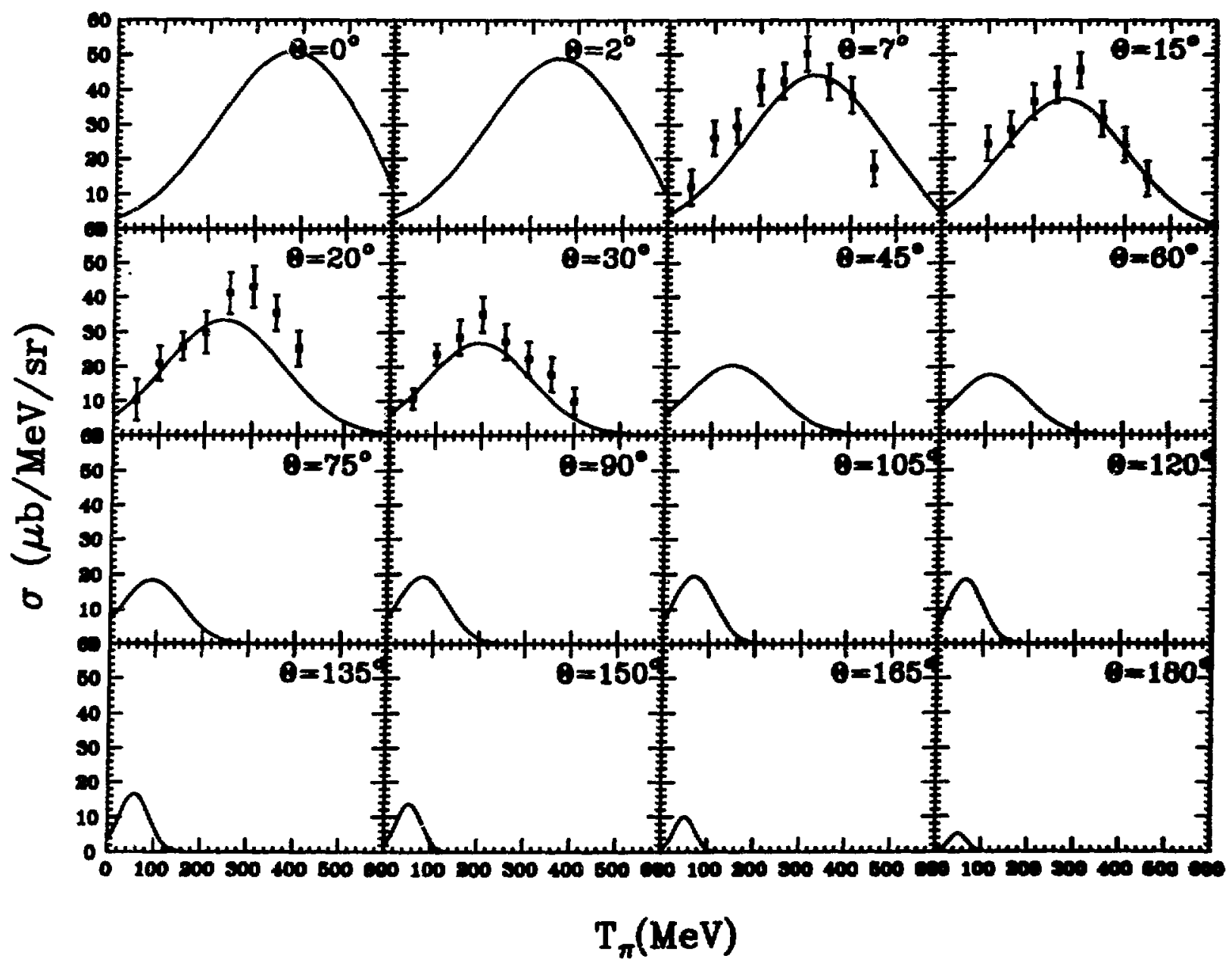

Fig. 9. The parameterization for $\pi^{+}$production from carbon is compared with the data from Ref. 8. These data are a test of the parameterization, as they were not used to fix any parameters. 
Inclusive $\pi^{+}$Production from Copper $\left(\mathrm{T}_{\mathrm{p}}=585 \mathrm{MeV}\right)$

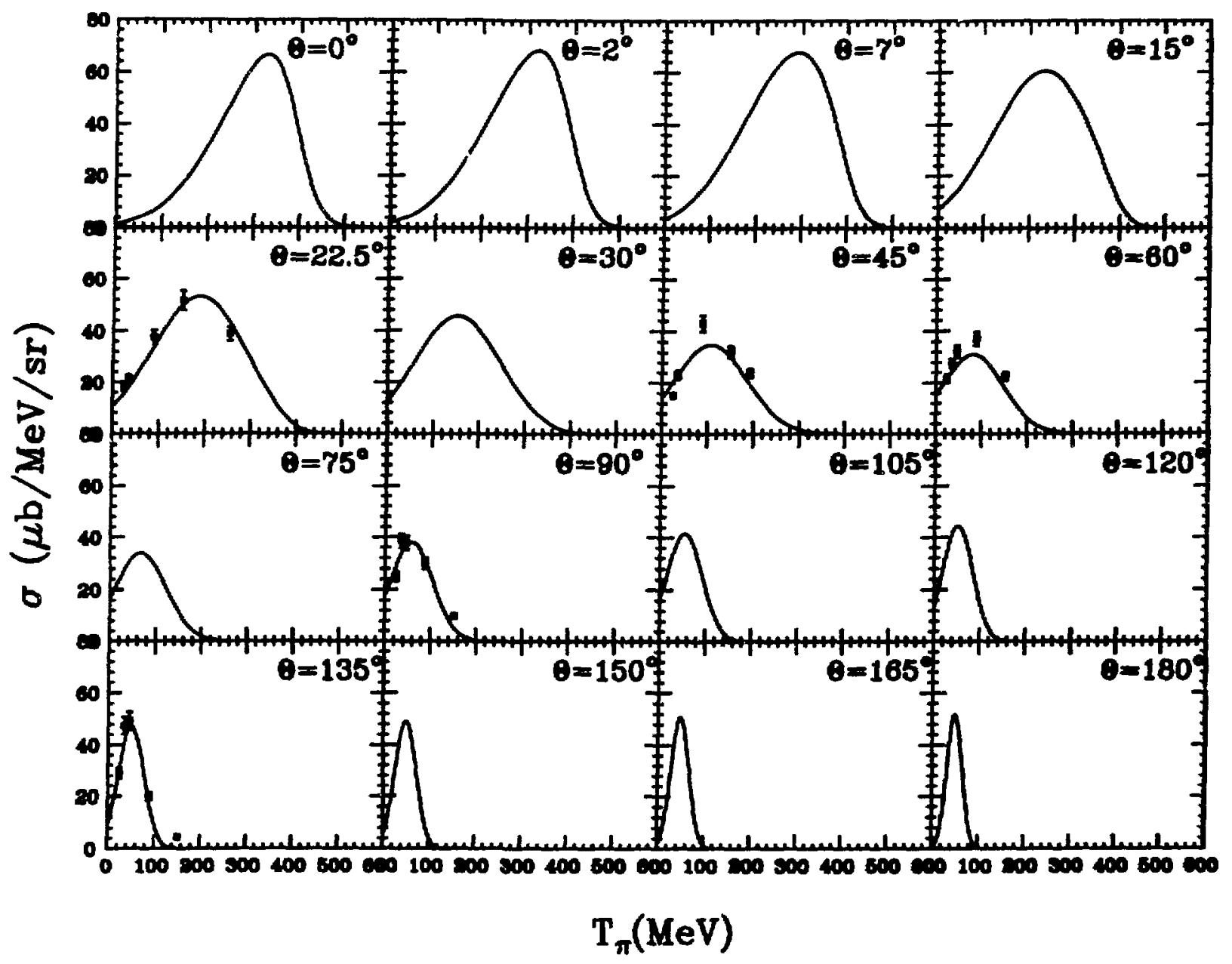

Fig. 10. The parameterization for $\pi^{+}$production from copper is compared with the data from Ref. 4. 


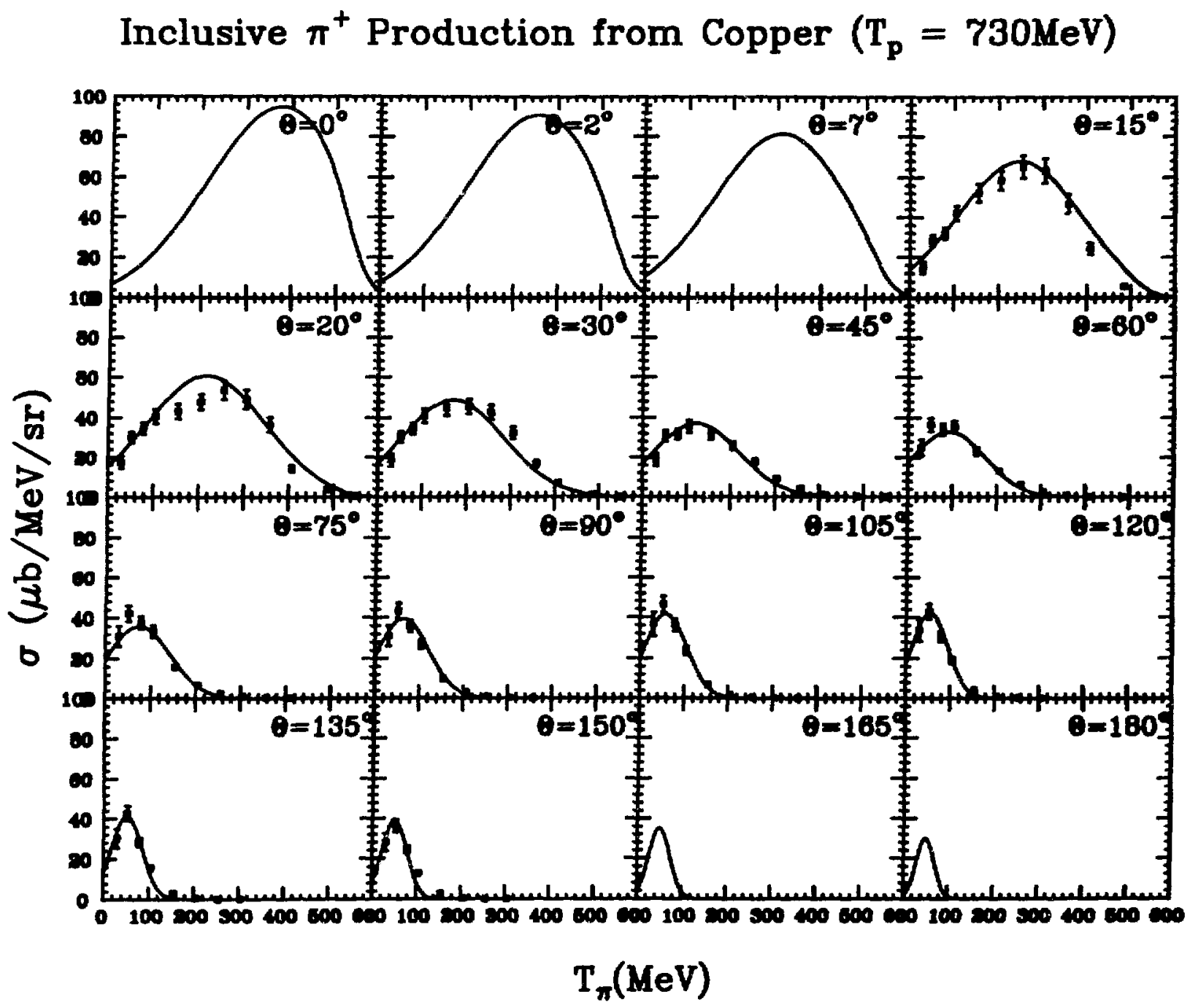

Fig. 11. The parameterization for $\pi^{+}$production from copper is compared with the data from Ref. 3. 
Inclusive $\pi^{+}$Production from Lead $\left(\mathrm{T}_{\mathrm{p}}=585 \mathrm{MeV}\right)$

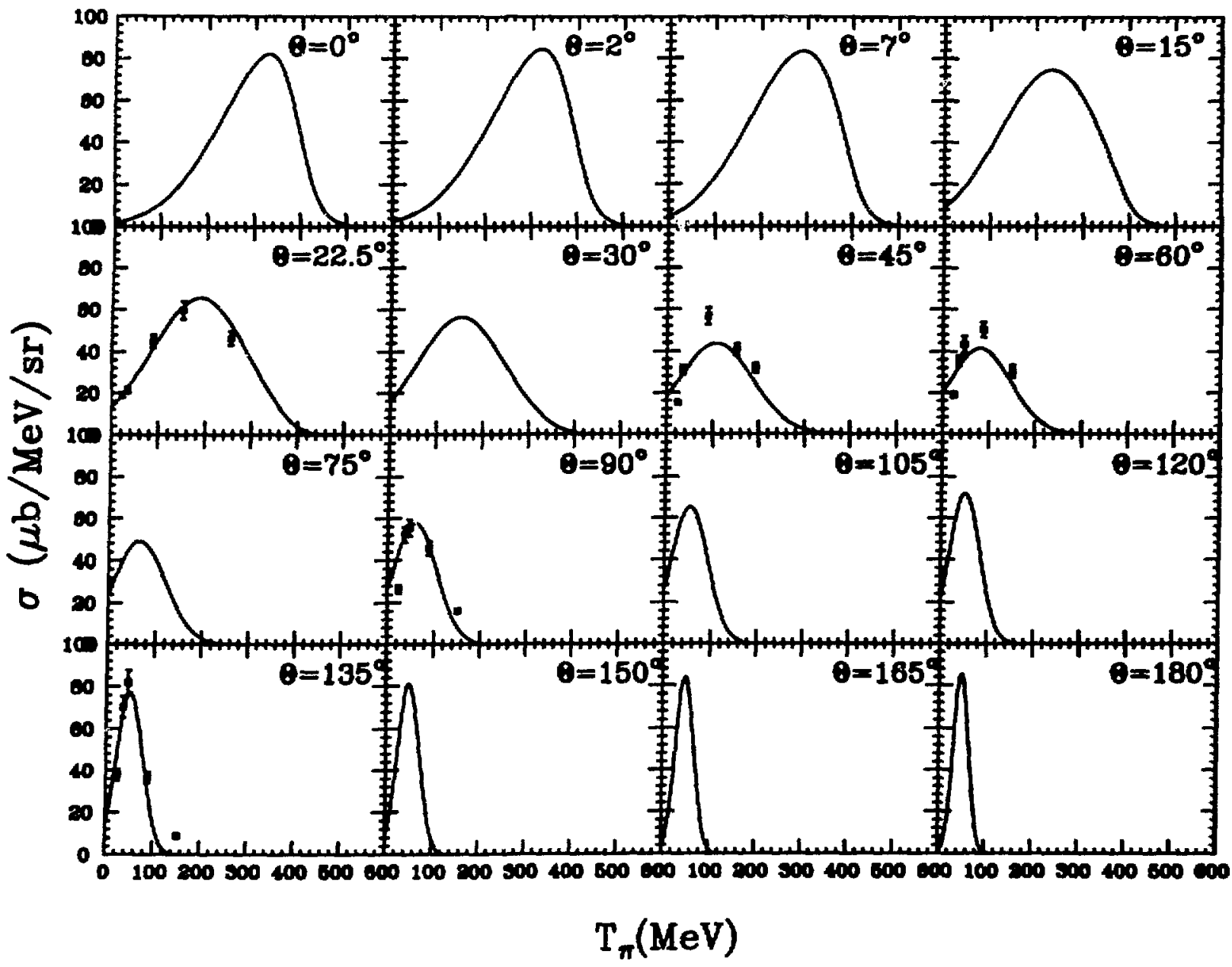

Fig. 12. The parameterization for $\pi^{+}$production from lead is compared with the data from Ref. 4. 
Inclusive $\pi^{+}$Production from Lead $\left(T_{p}=730 \mathrm{MeV}\right)$

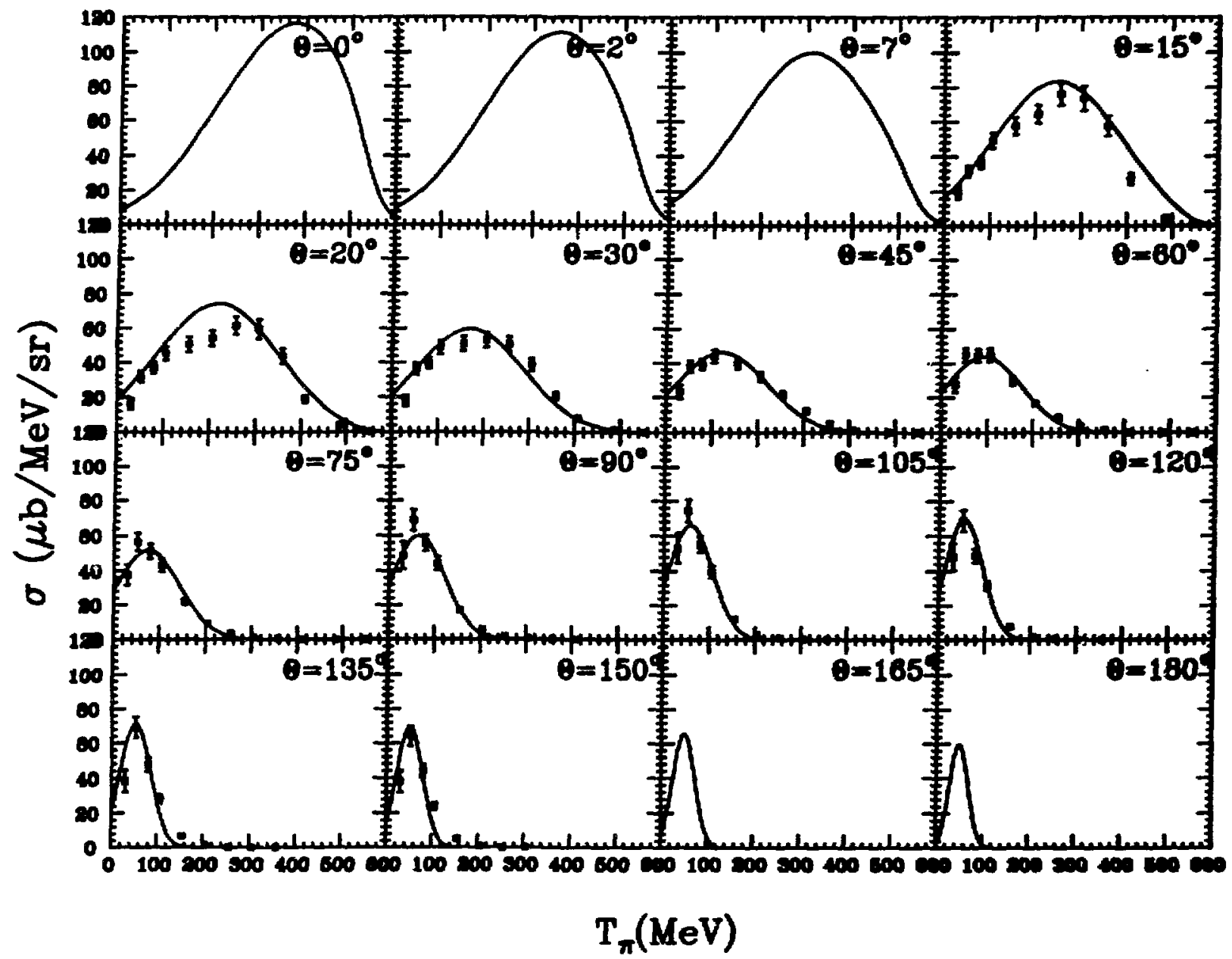

Fig. 13. The parameterization for $\pi^{+}$production from lead is compared with the data from Ref. 3. 
$30^{\circ}$ and does not change the pion energy appreciably. It is, therefore, unlikely that this process can change the distribution of pions stopping in the beam stop, so we do not treat this process in detail.

We define the pion-reaction cross section as the sum of inelastic scattering and absorption cross sections:

$$
\sigma_{\text {reac }}=\sigma_{\mathrm{abs}}+\sigma_{\text {inelastic }}
$$

The absorption cross section $\sigma_{\text {abs }}$ includes both true pion absorption and single charge exchange, where the $\pi^{+}$is converted into a $\pi^{0}$. Because the $\pi^{0}$ does not produce neutrinos and decays almost instantaneously, its effect is the same as true absorption. As a note, we might add that the experiments measure the sum of true absorption and charge exchange. The true absorption cross sections are obtained by subtracting the single charge exchange, typically about $15 \%$ of the sum.* The inelastic cross section $\sigma_{\text {inelastic }}$ can be obtained in two ways. Both methods rely on calculations of the elastic cross section that are subtracted from either measured scattering rates or the total cross section. ${ }^{15,16}$

We have choosen to parameterize the reaction cross section, as it is determined solely from the total and elastic cross sections. We use the usual form ${ }^{7}$

$$
\sigma_{\text {reac }}=\sigma_{0}\left(T_{\pi}\right) A^{\alpha\left(T_{\pi}\right)}
$$

where $A$ is the atomic weight and $\sigma_{0}$ and $\alpha$ are both functions of the pion energy $T_{\pi}$. For maximum flexibility we parameterize $\sigma_{0}$ and $\alpha$ with $B$-splines:

$$
\begin{array}{rlr}
\text { Exponent : } \quad \alpha=a_{1} & \left(0 \leq T_{\pi} \leq 50 \mathrm{MeV}\right) \\
\text { or } \quad \alpha \sum_{n=1}^{5} a_{n} B_{n} & \left(50 \leq T_{\pi} \leq 600 \mathrm{MeV}\right) \\
\text { where } \quad a_{1}=1.023 & \\
a_{2} & =0.816 \\
a_{3} & =0.501 \\
a_{4} & =0.715 \\
a_{5} & =0.670
\end{array}
$$

Spline knot sequence $=(50,50,50,120,250,600,600,600 \mathrm{MeV})$

* Double-charge-exchange cross sections are an order of magnitude smaller than singlecharge-exchange cross sections and are, therefore, not treated here. 


$$
\text { Normalization : } \begin{aligned}
\sigma_{0} & =\sum_{n=1}^{0} a_{n} B_{n} \\
a_{1} & =0 \\
a_{2} & =1.993 \\
a_{3} & =139.753 \\
a_{4} & =65.142 \\
a_{5} & =39.328 \\
a_{6} & =33.000
\end{aligned}
$$

Spline knot sequence $=(0,0,0,120,250,350,600,600,600 \mathrm{MeV})$.

The parameterizations are plotted in Fig. 14. We have extended the parameterizations smoothly beyond the measurements by requiring that the exponent be approximately one below $T_{\pi}=50 \mathrm{MeV}$ and $2 / 3$ at $600 \mathrm{MeV}$. At lower energies, the shape of the normalization $\sigma_{0}$ is dominated by the $(3,3)$ resonance. At higher energies we extend the parameterization slowly downward in a shape similar to that measured for the $\pi^{+} p$ total cross section.

The decomposition of the reaction cross section into $\sigma_{\mathrm{abs}}$ and $\sigma_{\text {inelastic }}$ is given by ${ }^{\mathbf{1 7}}$

$$
\begin{aligned}
\frac{\sigma_{\text {abs }}}{\sigma_{\text {reac }}} & =\sum_{n=0}^{2} c_{n}(\ln A)^{n} \\
c_{0} & =0.2927 \\
c_{1} & =0.0246 \\
c_{2} & =0.0074
\end{aligned}
$$

The decomposition is for $T_{\pi}=165 \mathrm{MeV}$ (Fig. 15); however, we use it at all energies. This simplification constrains the shapes of $\sigma_{\mathrm{abs}}$ and $\sigma_{\text {inelastic }}$ to be the same as a function of $T_{\pi}$. There is no a priori reason for this to be the case, but on the average it seems to represent the data (see Fig. 16). No clear trends are evident that might be used to construct a different parameterization.

The general features of inelastic pion scattering from nuclei are consistent with quasifree scattering. ${ }^{18,19,20}$ The peak in the energy spectrum of the scattered pion follows the kinematics of free $\pi^{+} p$ kinematics; its width is consistent with Fermi broadening. Even in heavy nuclei, multiple scattering processes are less than hali of the summed inelastic cross section at $163 \mathrm{MeV}$. The angular distributions vary with energy, but are similar to $\pi^{+} p$ elastic scattering distributions. Since, in the Monte Carlo program, we integrate over both energy and angle, we assume a simple $s$ - and $p$-wave distribution with the $p$-wave coefficient equal to $5 / 6$ and the $s$-wave coefficient equal to $1 / 6$. We then impose the kinematics of free-nucleon scattering. 


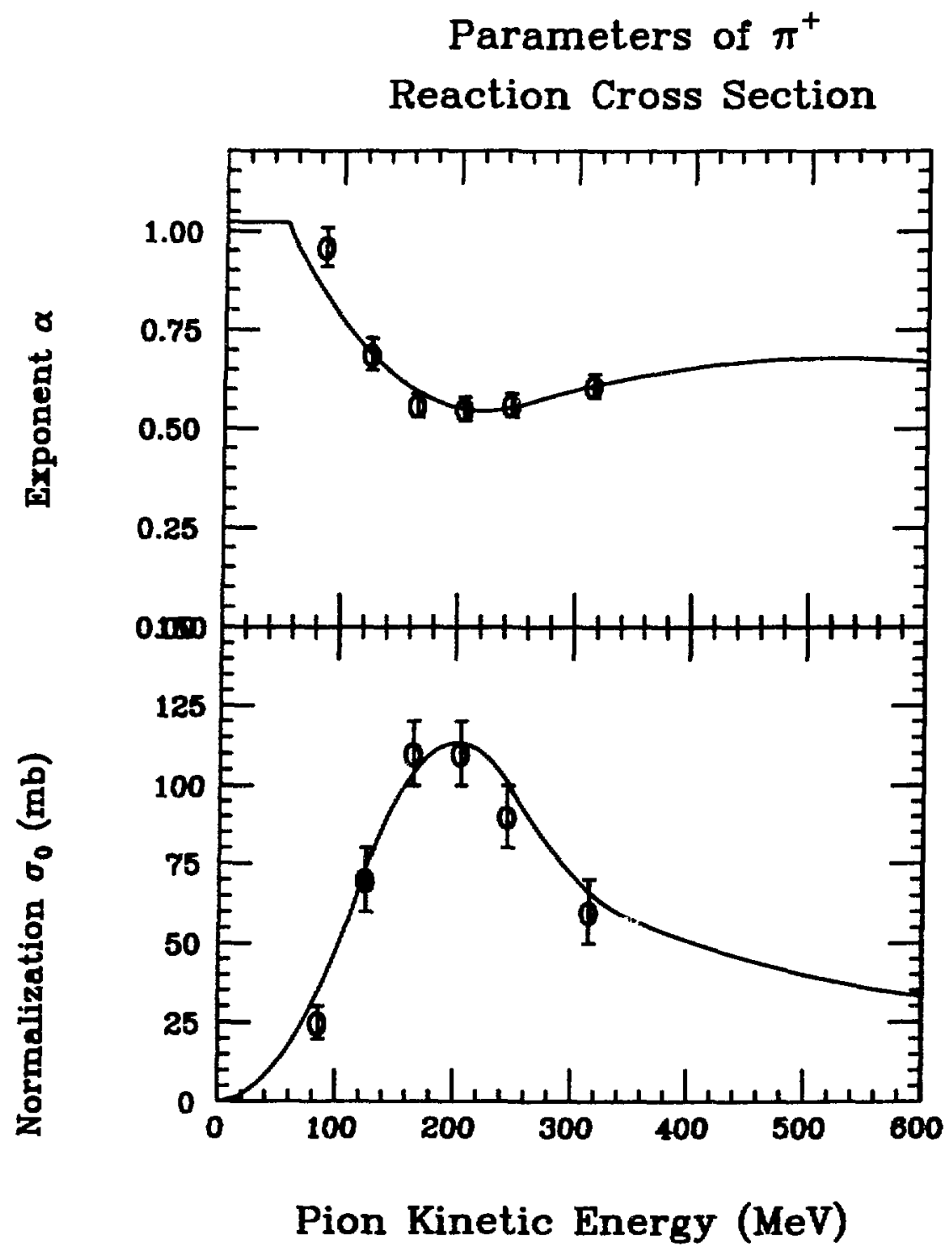

Fig. 14. The parameterization is shown for the normalization and exponent of the $\pi^{+}$reaction cross section, which is taken to be of the form $\sigma_{\text {reac }}=\sigma_{0} A^{\alpha}$. The data points are fits determined in Ref. 7. 


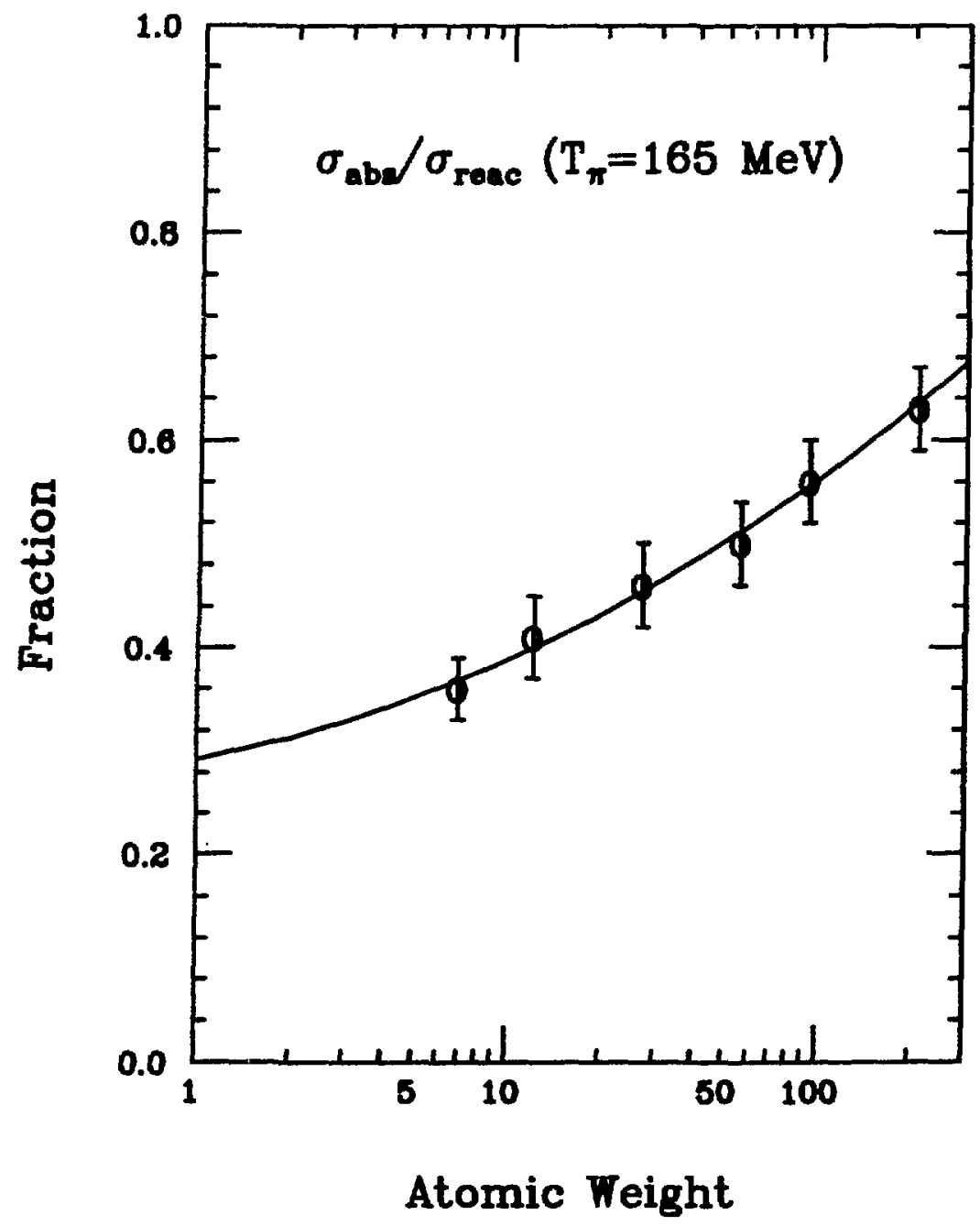

Fig. 15. The decomposition of $\sigma_{\text {reac }}$ into $\sigma_{\mathrm{abs}}$ and $\sigma_{\text {inelastic }}$ is parameterized at $T_{\pi}$ $=165 \mathrm{MeV}$. The values for the ratio are taken from Ref. 7 . 


\section{Pion Reaction Cross Sections $\left(\pi^{+}\right)$ \\ (mb)}

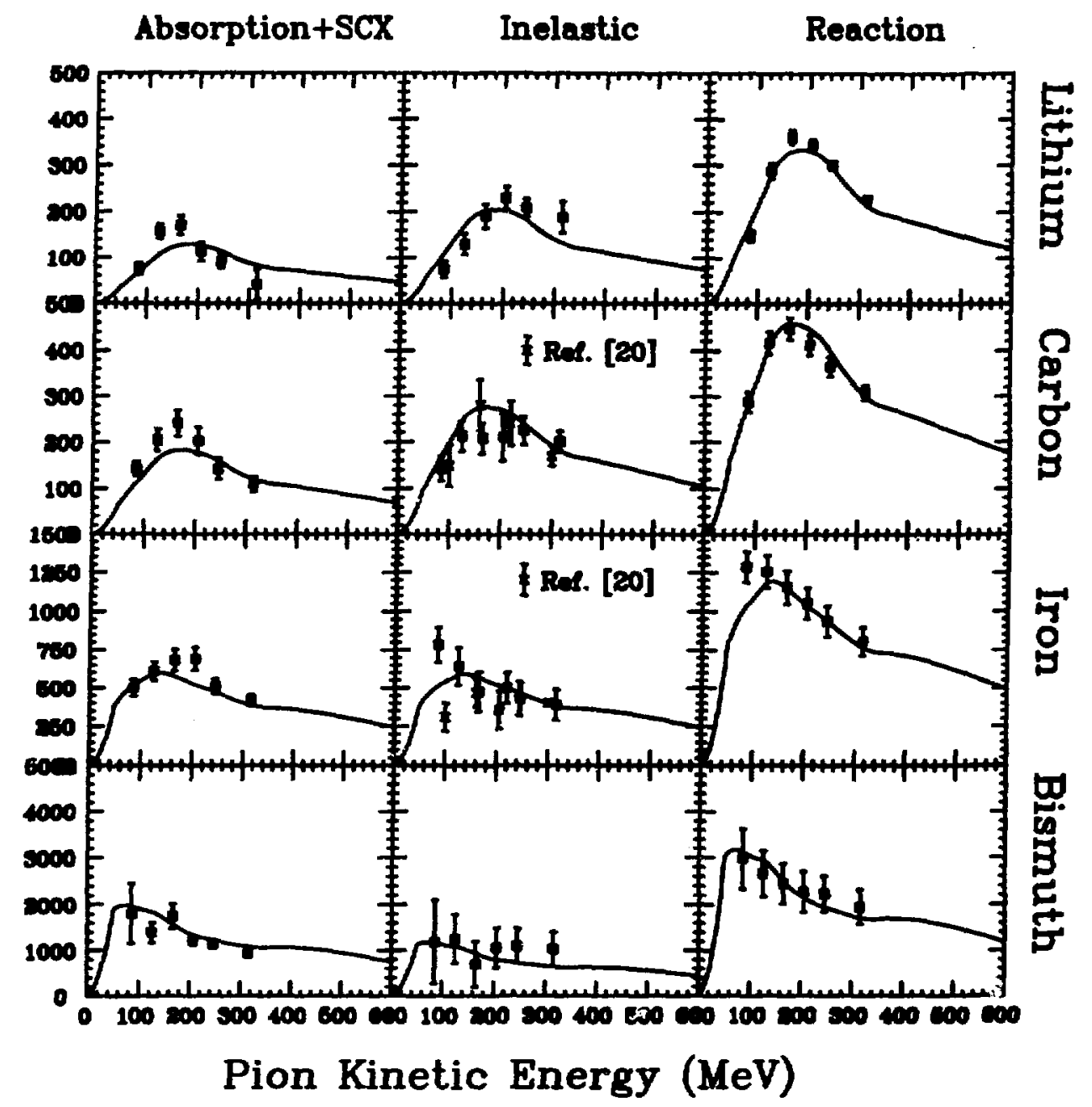

Fig. 16. Comparison of the parameterization of $\sigma_{\text {reac }}$ with the data from Ref. 16, except where noted.

\section{SUMMARY}

The prediction of the neutrino flux from the decay of stopping pions produced from proton interartions in the LAMPF beam stop requires extensive knowledge of pion production and pion reaction cross sections. Theoretical models do not predict these cross sections in enough detail to be used for the flux calculation. We have, therefore, turned to representations of the data, which are well-behaved and can be extrapolated smoothly to regions where there are no measurements. This has been achieved either through linear 
approximations or parabolic spline representations of parameters that describe the cross sections.

LAMPF Experiment $866^{1}$ has measured the distribution of pions stopping inside a mockup beam stop. The parameterization described in this paper will be used to reproduce these data by tuning parameters that are not constrained by the measured pion cross sections. This will be accomplished by first determining the sensitivity of the stopping pion distributions to the overall cross-section normalizations. Further differences will be resolved by adjusting the parameters of the pion-reaction cross section, which are poorly measured in relation to the cross sections for pion production.

\section{REFERENCES}

1. R. Allen and J. B. Donahue, spokespersons, "Calibration of the Neutrino Flux at the LAMPF Beam Stop," LAMPF Research Proposal 866.

2. R. L. Burman, M. E. Potter, and E. S. Smith, "A Monte Carlo Simulation of Neutrino Production at a Medium-Energy Proton Beam Stop" (in preparation for Nucl. Instr. and Meth.) (1989).

3. D. R. F. Cochran, P. N. Dean, P. A. M. Gram, E. A. Knapp, E. R. Martin, D. E. Nagle, R. B. Perkins, W. J. Shlaer, H. A. Thiessen, and E. D. Theriot, Phys. Rev. D6, 3085 (1972).

4. J. F. Crawford, M. Daum, G. H. Eaton, R. Prosch, H. Hirschmann, R. Horisberger, J. W. McCulloch, E. Steiner, R. Hausammann, R. Hess, and D. Werren, Phys. Rev. C22, 1184 (1980).

5. Newsline, The Journal of Nuclear Medicine 28, 1371 (1987).

6. R. Maschuw, "Search for Neutrino Oscillations with KARMEN," in Proceedings of the Sixth Moriond Workshop on Massive Neutrinos in Astrophysics and in Particle Physics, 1986, O. Fackler and J. Trân Thanh Vân, Eds. (Editions Frontières), p. 293 (1986).

7. Daniel Ashery and John P. Schiffer, Ann. Rev. Nucl. Part. Sci. 36, 207 (1986).

8. Peter Denes, B. D. Dieterle, D. M. Wolfe, T. Bowles, T. Dombeck, J. E. Simmons, T. S. Bhatia, G. Glass, and W. B. Tippens, Phys. Rev. C27, 1339 (1983).

9. F. H. Cverna, P. R. Bevington, M. W. McNaughton, H. B. Willard, N. S. P. King, and D. R. Giebink, Phys. Rev. C23, 1698 (1981).

10. See references in Ref. 4.

11. T. A. Dombeck, "LANLNU-A Description of the Monte Carlo Computer Calculation of the Neutrino Flux at the High-Intensity Los Alamos Neutrino Source," Los Alamos National Laboratory document LA-UR-82-1589 (1982).

12. Common Los Alamos Mathematical Software Compendium pp. 5-39 (1984). The routines used were adapted from C. de Boor, A Practical Guide to Splines (Springer-Verlag, New York, 1978).

13. B. J. VerWest and R. A. Arndt, Phys. Rev. C25, 1979 (1982).

14. J. F. Marshall, M. E. Nordberg, and R. L. Burman, Phys. Rev. C1, 1685 (1970).

15. I. Navon, D. Ashery, G. Azuelos, H. J. Pfeiffer, H. K. Walter, and F. W. Schlepütz, Phys. Rev. Lett. 42, 1465 (1979). 
16. D. Ashery, I. Navon, G. Azuelos, H. K. Walter, H. J. Pfeiffer, and F. W. Schlepütz, Phys. Rev. C23, 2173 (1981).

17. J. P. Schiffer, Nucl. Phys. A335, 339 (1980).

18. C. H. Q. Ingram, Nucl. Phys. A374, 319 (1982).

19. S. M. Levenson, D. F. Geesaman, E. P. Colton, R. J. Holt, H. E. Jackson, J. P. Schiffer, J. R. Specht, K. E. Stephenson, B. Zeidman, R. E. Segel, P. A. M. Gram, and C. A. Goulding, Phys. Rev. Lett. 47, 479 (1981).

20. S. M. Levenson, D. F. Geesaman, E. P. Colton, R. J. Hoit, H. E. Jackson, J. P. Schiffer, J. R. Specht, K. E. Stephenson, B. Zeidman, R. E. Segel, P. A. M. Gram, and C. A. Goulding, Phys. Rev. C28, 326 (1983).

21. N. J. DiGiacomo, M. R. Clover, R. M. DeVries, J. C. Dousse, J. S. Kapustinsky, P. L. McGaughey, W. E. Sondheim, J. W. Sunier, M. Buenerd, and D. Lebrun, Phys. Rev. C31, 292 (1985).

22. L. Bimbot, V. Bellini, M. Bolore, X. Charlot, C. Guet, J. M. Hisleur, J. C. Jourdain, J. Julien, P. Kristiansson, G. Lanzano, B. Million, A. Oskarsson, A. Palmeri, G. S. Pappalardo, J. Poitou, F. Reide, and N. Willis, Nucl. Phys. A440, 636 (1985). 